\title{
Basal Forebrain Lesioned Mice Exhibit Deterioration in Memory Acquisition Process in Step Through Passive Avoidance Test
}

\author{
Akane Ishihara, Hiroshi Saito, Hiroyuki Ohta and Nobuyoshi Nishiyama* \\ Department of Chemical Pharmacology, Faculy of Pharmaceutical Sciences, University of Tokyo, \\ Bunkyo-ku, Tokyo 113, Japan
}

Received January 21, 1991 Accepted July 18, 1991

\begin{abstract}
Effects of the basal forebrain (BF) lesion on memory and learning performances were investigated in mice. Eight-week-old male mice received bilateral $\mathrm{BF}$ lesion by delivering a radiofrequency current. From fifteen days after the surgery, the step through type passive avoidance task was performed daily for 10 days. Lesioned animals showed severe impairment in the acquisition process of this task, but not in the retention process. Ambulatory activity of the BF-lesioned mice did not differ from those of the control group, suggesting the observed learning impairment was not due to the alteration of motor activity. These results indicate that a memory impaired model mice can be successfully made by the radiofrequency lesion of bilateral BF neurons.
\end{abstract}

Alzheimer's disease has a characteristic pattern of brain degeneration that is associated with a progressive impediment of cognitive functions. There are specific neuropathological changes that include neurofibrillary tangles, senile plaques, and degeneration of specific nerve cells $(1-4)$. The reduction of presynaptic cholinergic markers such as choline acetyltransferase (ChAT) activity in the neocortex has been observed in Alzheimer's discase patients. This reduction is correlated with loss of cholinergic cells in the basal forebrain (BF) and the degree of dementia in clinical studies $(5-8)$. The $\mathrm{BF}$ cholinergic system has also been shown to play an important role in learning and memory function in experimental animals; and using rats, many investigators have found that lesioning of this region causes severe deficits in various kinds of learning

\footnotetext{
* To whom correspondence should be addressed.
}

tasks $(9-15)$. In the present study, for the purpose of making a new murine model which can be more easily used for the screening of antidementic drugs, the effects of BF lesion on passive avoidance performances were investigated in mice.

\section{MATERIALS AND METHODS}

\section{Animals}

Malc Std-ddY mice weighing $35-39 \mathrm{~g}, 8$ weeks old at the start of experiments, were used (SLC, Hamamatsu, Japan). The animals were housed in groups of 5 in metal cages (30 $\times 20 \times 10 \mathrm{~cm})$ in a temperature- $\left(22 \pm 1^{\circ} \mathrm{C}\right)$ and humidity- $(55 \pm 5 \%)$ controlled room. Food (CE-2, Clea Japan, Inc., Tokyo, Japan) and water were available ad libitum.

\section{BF lesion}

Under anesthesia with a ketamine and xyla- 
zine mixture ( 80 and $7 \mathrm{mg} / \mathrm{kg}$, respectively, i.m.), mice were placed on a stereotaxic apparatus. They received bilateral $\mathrm{BF}$ lesion by delivering a radiofrequency current (LESION, $\mathrm{n}=15$; using a lesion generator, Radionics Inc., Burlington, U.S.A.). The coordinates of the electrode placements used were: $0.8 \mathrm{~mm}$ posterior to the bregma, \pm 2.8 $\mathrm{mm}$ lateral to the sagittal suture, and $4.3 \mathrm{~mm}$ below the dura, with the incisor bar placed at $2.2 \mathrm{~mm}$ ventral to the horizontal plane. The stainless steel electrode $(0.25 \mathrm{~mm}$ diameter $)$ was inserted in the brain, and the tip of the electrode was heated at $70^{\circ} \mathrm{C}$ for $60 \mathrm{sec}$. For the sham-operated mice (SHAM, $\mathrm{n}=15$ ), the inserted clectrode was not heated. Mice given only the anesthetic were served as the control group (CONT, $\mathbf{n}=15$ ). After the operation, commercial milk was given to the lesioned mice twice a day for a week (total $2 \mathrm{ml} /$ day, p.o.). Fifteen days after surgery, when the body weights of the lesioned mice were recovered, they were submitted, according to Segawa's method (16), to two passive avoidance type learning tasks (step through and step down tests) and motor activity measurement with a tilting-type ambulometer ( $O^{\prime}$ Hara \& Co., Ltd., Tokyo, Japan) for $30 \mathrm{~min}$.

\section{Behavioral tests}

In the step through test, a two-compartment step-through type passive avoidance apparatus (PA M1, O'Hara \& Co., Ltd., Tokyo, Japan) was used. In the acquisition trial, a mouse was placed in the front illuminated chamber. When it entered the rear darkened compartment (error), a $36-\mathrm{V}$ footshock $(\max 0.2 \mathrm{~mA}$ ) was applied through the grid floor. The time that elapsed before the mouse entered the dark compartment was recorded. From $24 \mathrm{hr}$ after this acquisition trial, the mice were submitted to a testing session that consisted of a daily testing trial for ten days at the same time of day. During the testing session, the punishing footshock was always given to the mice that stepped through into the dark compartment. In the first testing trial, a mouse was replaced in the light compartment, and the latency to enter the dark compartment was measured with the ceiling score of $300 \mathrm{sec}$. This ceiling score of $300 \mathrm{sec}$ was regarded as a criterion, and a mouse that did not enter the dark compartment for $300 \mathrm{sec}$, that is, a mouse that did not receive the punishing electro-shock for the first time, was regarded to have reached the criterion. The days taken to reach this criteria ("Mcmory Acquisition") were measured as a parameter of memory acquisition abilities. The mean days during which the mice made no error after they had once attained the criteria ("Memory Retention") were measured as memory retention abilities.

The apparatus of the step down test consisted of a chamber equipped with a grid floor with an escaping platform located in the corner of the chamber. In the acquisition trial, a mouse was placed on the platform and allowed to explore for $10 \mathrm{~min}$. When the mouse got off from the platform, a $50-\mathrm{V}$ footshock was applicd through the floor grid. During the latter half of the acquisition trial, the number of shocks received by descent and the number of descended mice were counted. From $24 \mathrm{hr}$ after this acquisition trial, the mice were submitted to a testing session that consisted of a daily testing trial for ten days at the same time of day. During the testing session, the punishing footshock was always given to the mice that stepped down. In the first testing trial, the mouse was placed on the platform, and the number of descents were counted for $3 \mathrm{~min}$. No descent from the platform for $3 \mathrm{~min}$ was regarded as the criterion, and the same parameters as the step through test were measured.

\section{Histological studies}

After the behavioral tests, at 31 days after surgery, the brain was removed, fixed with Bouin's solution and sectioned coronally at 50 $\mu \mathrm{m}$ thickness with a frozen-stage microtome and stained with hematoxyline-eosin (HE). The location of the lesioned site was assessed by microscopic examination. The volume of the lesioned area was measured using an 
image analyzer (MGA-1300, Mutoh Kohgyo, Japan).

\section{Statistics}

Mann-Whitney's $U$-test was used for statistical analysis of the mean latency, the mean number of descents, memory acquisition and retention. The chi-square test was used to analyze the percent of mice without error. Analysis of variance (ANOVA) followed by Duncan's test was used for motor activity.

\section{RESULTS}

The body weights of the operated mice decreased from soon after the surgery for 3 days. After that, the mice began to regain weight, and almost all the mice recovered their weight in 15 days (Fig. 1). However, 1 animal in the SHAM group and LESION group, respectively, were omitted from the further experiments because they had ncck torsion and abnormal hyperactivity.

The lesioned sitc was located in the ventromedial region of the globus pallidus, which correspond to the rodent's BF (Fig. 2). The average volume of the lesioned area was 0.83 $\pm 0.05 \mathrm{~mm}^{3}$. In the lesioned area, gliosis was observed.

Motor activity measured with the tilting type ambulometer is shown in Fig. 3. Animals in the SHAM and CONT groups tended to exhibit slightly higher spontaneous motor activity in the later days, while the lesioned mice kept a constant level. However, the difference among the three groups was not significant throughout the testing period.

Figure 4 shows the results of the step through test. The mean latency to enter the dark chamber in the acquisition trial was not different among the groups, while in the first testing trial, the latency in the LESION group was significantly shorter than those in the SHAM and CONT groups (Fig. 4a). Figure 4b shows the percentage of mice that did not enter the dark compartment in the first testing trial. In the CONT and SHAM groups, 73 and $92 \%$ of the mice, respectively, stayed in the lightened compartment for $300 \mathrm{sec}$ on the first testing trial. In contrast, $85 \%$ of the lesioned mice entered the dark compartment within 300 sec. Furthermore, lesioned mice took signifi-

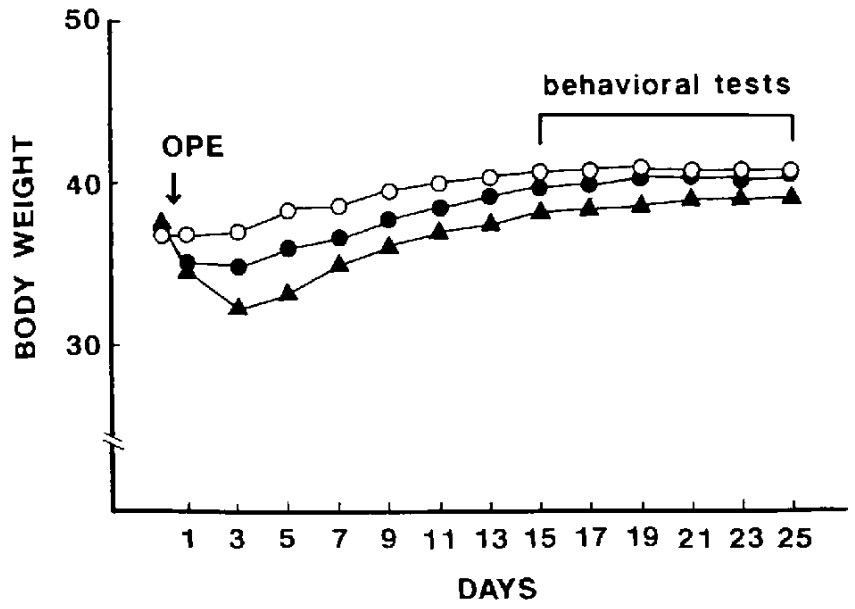

Fig. 1. Change in the body weight after bilateral BF lesion in mice. $\bigcirc$ : anesthetization only (CONT), sham-operated (SHAM), A : bilateral BF lesion by delivery of a radiofrequency current (LESION). The arrow indicates the day of BF lesion, and the horizontal axis represents the day after the operation. The bar indicates the period during which behavioral tests were performed. In this period, body weights were measured before the behavioral tests. 


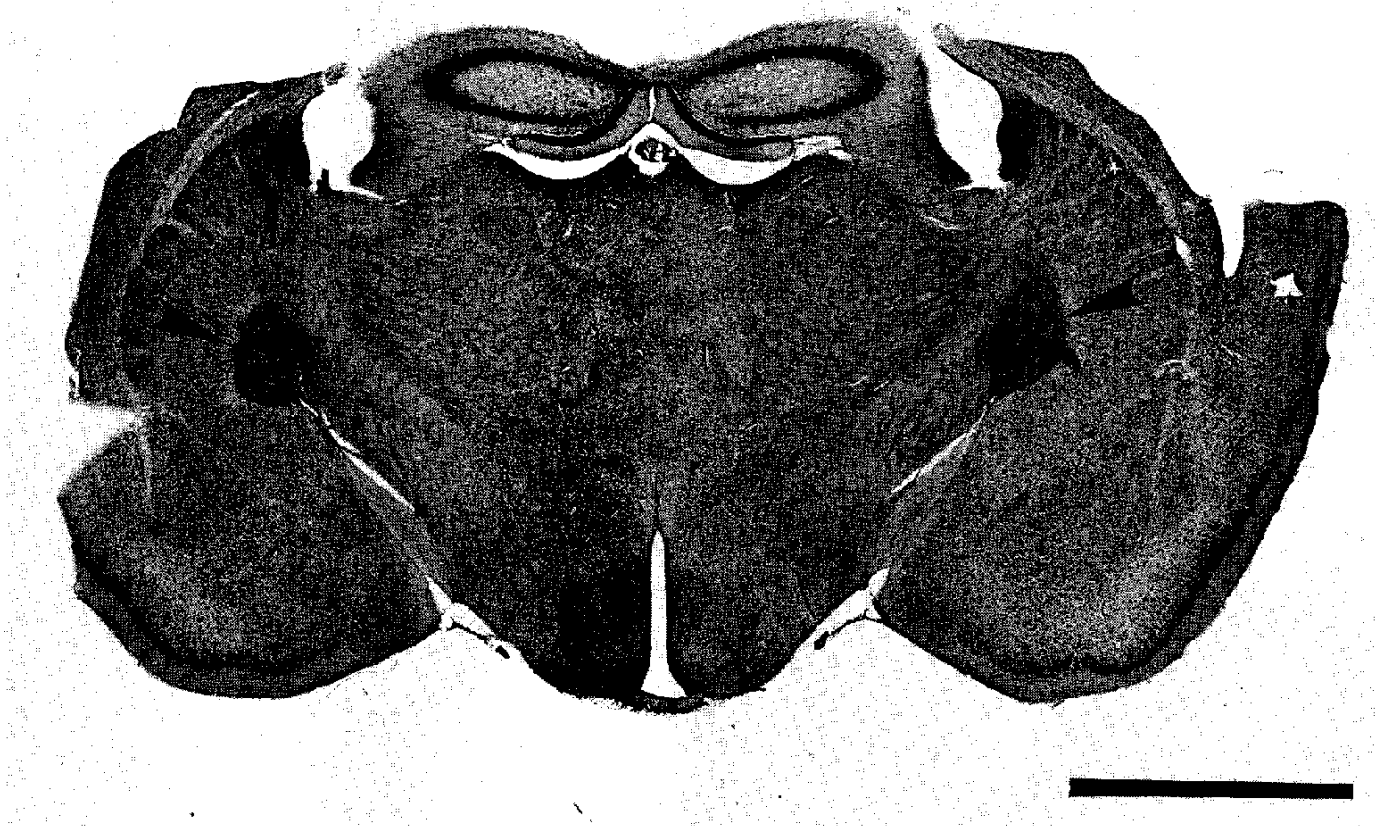

Fig. 2. Histological visualization of the lesioned area. Fifty- $\mu \mathrm{m}$ frozen sections were stained with HE 31 days after the operation. The cerebral cortex was removed. The bar represents $2 \mathrm{~mm}$, and the arrowheads indicate the lesioned area.

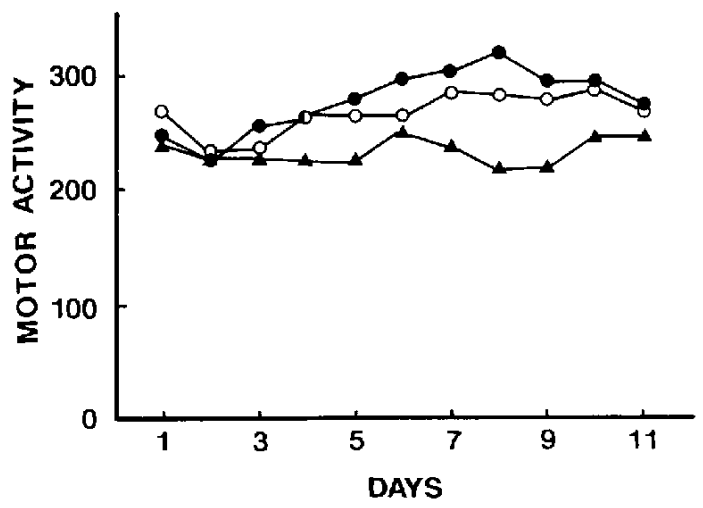

Fig. 3. Motor activity during the behavioral testing. O: CONT, O: SHAM, A: LESION. See the legend to Fig. 1 for explanations. Motor activity was measured with a tilting-type ambulometer for $30 \mathrm{~min}$ before the passive avoidance tests. No significant difference was seen between the three groups. cantly more trials $(3.0 \pm 0.7)$ to reach the criteria than CONT and SHAM mice $(1.3 \pm$ 0.2 and $1.2 \pm 0.2$, respectively: Table 1$)$, indicating that the early memory process(es) was disturbed by BF lesion. On the other hand, LESION mice exhibited little or no deterioration of memory retention abilities (Table 1 ). Lesioned mice retained memory for $2.7 \pm 0.6$ days after having attained the critcria, which tended to be shorter than those of the CONT and SHAM groups, but the difference was not significant.

In contrast to the step through test, BF lesion resulted in no memory impairment in the step down test both in the acquisition and retention processes. In the first testing trial, percentages of mice without descents did not differ significantly among CONT, SHAM and 
(a)

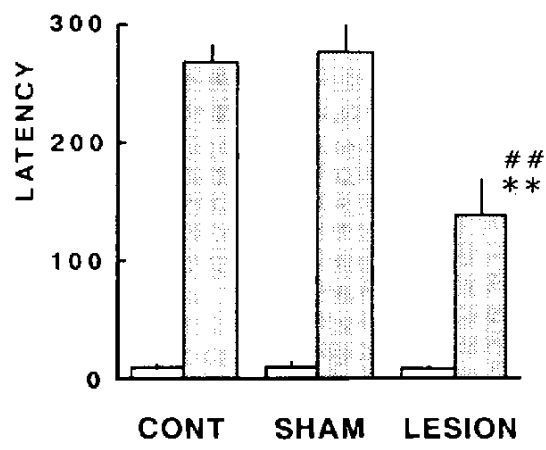

(b)

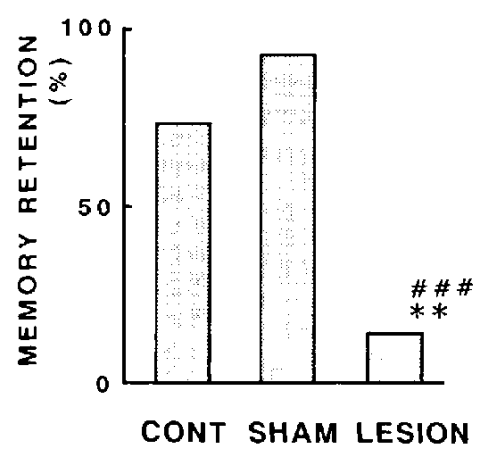

Fig. 4. Effect of bilateral BF lesion on the acquisition process in the step through test. (a) Mean latency to enter the dark chamber during acquisition (open column) and the first testing trial (dotted column) in the step through test. The bars represent S.E. \#\# $\mathrm{P}<0.01$ vs. SHAM, ${ }^{* *} \mathrm{P}<0.01$ vs. CONT, Mann-Whitney's $U$-test. (b) Percentage of mice that did not enter the dark compartment in the first testing trial. \#\#\#: $\mathrm{P}<0.001$ vs. SHAM, ${ }^{* *}: \mathrm{P}<0.01$ vs. CONT, chi square-test. See the legend of Fig. 1 for abbreviations.

Table 1. Memory acquisition and memory retention in step through and step down passive avoidance tests

\begin{tabular}{llll}
\hline & & Acquisition & Retention \\
\hline \multirow{3}{*}{ ST } & CONT & $1.3 \pm 0.2$ & $4.4 \pm 1.0$ \\
& SHAM & $1.2 \pm 0.2$ & $4.0 \pm 0.9$ \\
& LESION & $3.0 \pm 0.7^{\# \# *}$ & $2.7 \pm 0.6$ \\
SD & CONT & $1.3 \pm 0.1$ & $5.1 \pm 1.0$ \\
& SHAM & $1.6 \pm 0.2$ & $3.2 \pm 0.7$ \\
& LESION & $1.8 \pm 0.4$ & $3.6 \pm 0.7$ \\
\hline
\end{tabular}

Memory acquisition and retention indicate the mean days until the mice reached the criteria and the mean days when the mice made the first error after they attained this criteria, respectively. Results are given as means \pm S.E. [days]. ST: step through test, SD: step down test. For other abbreviations, see the legend to Fig. 1. ${ }^{\# \#: ~} \mathrm{P}<0.01$ vs. SHAM, ${ }^{* *}: \mathrm{P}<0.01$ vs. CONT, Mann-Whitney's $U$-test.

LESION groups (data not shown). Memory acquisition and retention was not affected by BF lesion (Table 1).

\section{DISCUSSION}

The basal forebrain cholinergic neurons are well know to play an important part in learning and memory function. Although many lesion experiments on this region have been per- formed in rats and monkeys $(9-15)$, little is known about mice. With the aim of making a BF lesion-induced amnesia model for the development of a new drug screening method, we chose mice as the experimental animal because they are easy to handle and require less drug. The radiofrequent (RF) lesion method was employed in this study, although neurotoxic lesion by ibotenic or kainic acid is the most commonly used procedure. Neurotoxins have an advantage because they selectively damage the cell body. Application of RF current is not specific for cholinergic cell bodies in the BF and destruction of the passage fibers through the globus pallidus is unavoidable. However, we felt that RF current would be more suitable to make a localized lesion than a diffusive toxin solution when small animals like mice are used. Even though the cholinergic specific neurotoxin AF64A is also used for hippocampal lesioning by intra-lateral-ventricular perfusion, few investigations have been done with this substance microinjected into BF nuclei.

Aphagia and adipsia were reported to occur acutely after BF lesioning; and in one study, it was reported to persist for up to 15 days (15). In the present experiment, animals recovered their body weight within 10 days, which also indicates the restricted damage of the lesioned 
area. Hepler et al. (17) compared the behavioral effects of $\mathrm{BF}$ lesion using neurotoxin and delivery of electric current and showed that they were qualitatively similar in the rat $T$ maze task. The participation of other transmitter systems or cholinergic neurons which are not included in the BF-cortical cholinergic systern may produce the quantitative difference.

The present study demonstrated that BF lesion disrupted the memory performances only in the step through test in mice. The step through latency was significantly shortened in the first testing trial and the avoidance ratio (percentage of mice with no errors) reduced in the LESION group. In repeated testing trials, the mean days before reaching the criteria also increased in the LESION mice, suggesting that $\mathrm{BF}$ lesion impaired the memory acquisition phase in this task. These data agreed with the previous reports of one- and multitrial passive avoidance tasks using rats $(9,10,12)$. Each mouse in the LESION group finally attained the criterion. On the other hand, the degree of memory retention were similar among the three groups. These indicate that after repeated training, lesioned animals can perform this task successfully and that they showed little, if any, memory impairment in the retention process, which is in agreement with some previous postlcsion-training studies $(18,19)$.

On the other hand, BF lesion after the acquisiton trial is reported to also reduce deficits in the retention process $(17,20)$. It is possible that by the end of the testing period, the memory function of the lesioned animals had already recovered because the animals used here were young adults that have neuronal plasticity. Bartus et al. (21) mentioned that functional recovery following $\mathrm{BF}$ lesion requires some degree of post-operative stimulation and task exposure. So sequential training in the step through and step down tests in identical animals may have hastened the functional recovery.

It is known that the decrease of shock sensitivity can be a cause of inferior abilities in avoidance tasks. Although animals were not evaluated quantitatively for alteration of shock sensitivity in this study, no behavioral differences were observed in their responses among the LESION, SHAM and CONT groups when the punishing electric shock was delivered from the grid floor of the passive avoidance apparatus. Furthermorc, there was no significant change in motor activity between the LESION group and other groups during the testing period. Motor hyperactivity in BFlesioned animals has been reported in some studies at two or three weeks after the operation $(15,22)$. In the present research, animals may have recovered from hyperkinesia, if any, by this time because of the limited damage. Taken together, the memory acquisition deficits observed in the step through task may not be due to the alteration in general behavior.

BF constitutes the principal source of cholinergic projections to the neocortex (23). Indeed, reductions of cholinergic markers such as high affinity choline uptake, ChAT and acetylcholine esterase activity were reported in BF-lesioned animals. We also found that choline acetyltransferase activity in the frontal cortex decreased about $20 \%$ in the lesioned mice compared to the control and sham-operated groups by 31 days after the operation (A. Ishihara et al., unpublished observation). This suggests that memory impairments in the step through test in the present study were at least partly duc to the lesion-induced reduction of cholinergic projections from the $\mathrm{BF}$ to the cerebral cortex

In contrast to the step through test, BF lesion did not induce significant changes in step down test performances. Very few investigations have been done using the step down test in BF-lesioned rats. Thus we cannot compare the species difference in step down performance in BF-lesioned animals. We have already certified that scopolamine, ethanol or electric shock impair both of these learning performances to the same extent under the identical conditions and apparatuses used in the present expcriment. Thus the task conditions of both the tests such as the intensity of the punishing 
electric shock or the size of the apparatus are partinent for screening. Our results indicate that those two passive avoidance tasks qualitatively differ from each other in some manner.

The present data, at the same time, may indicate that the degree of contribution of the BF-cerebral cortex cholinergic system is different in these two types of passive avoidance tasks. Segawa et al. $(16,24)$ reported that neurochemical parameters of the cholinergic system in discrete brain rcgions are differentially modulated by step through and step down tasks. Especially, acetylcholine contents and ChAT activity were increased and the number of muscarinic binding sites was decreased in the hippocampus of mice that were given the step down task, but not in those of step through trained animals. Thus the septohippocampal cholinergic system may play a more important role in the step down task than the BF-cortical cholinergic one. However, further experimental data are necessary to clarify these differences.

Non-cholinergic neurons and/or passage fibers through the globus pallidus were also destroyed by our lesioning method. The existence of neurons containing $\gamma$-aminobutyric acid in the BF magnocellular complex have been reported in rats and cats $(25,26)$. Also in primates, non-cholinergic neurons that contain neuropeptides are known to be intermingled among cholinergic cells of this area (27). Thus the contribution of other transmitter system(s) also should be considered.

In conclusion, bilateral $\mathrm{BF}$ lesion resulted in memory deficits in the acquisition process of the step through test in mice. This murine model of Alzheimer's disease can be used as a novel efficient screening method for antiamnesic drugs.

\section{REFERENCES}

1 Arendt, T., Baigl, V., Tennsted, A. and Arendt, A.: Neuronal loss in different parts of the nuclcus basalis is related to ncuritic plaque formation in cortical target areas in Alzhcimer's disease. Neuroscience 14, 1- 14 (1985)
2 Coyle, J.T., Price, D.L. and DeLong, M.R.: Alzheimer's discase: A disorder of cortical cholinergic innervation. Science 219, 1184-1190 (1983)

3 Davies, P. and Maloncy, A.J.F.: Selective loss of central cholinergic neurons in Alzhcimer's disease. Lancet 2, 1403 (1976)

4 Whitehouse, P.T., Price, D.L., Strublc, R.G., Clark, A.W., Coyle, J.T. and DeLong, M.R.: Alzheimer's disease and senile dementia: loss of neurons in the basal forebrain. Science 215, $1237-$ 1239 (1982)

5 McGeer, P.L., McGeer, E.G., Suzuki, J., Dolman, C.E. and Nagai, T.: Aging; Alzhemer's discase, and the cholinergic system of the basal forebrain. Neurology 34,741-745 (1984)

6 Perry, E.K., Gibson, P.H., Blessed, G., Perry, R.H. and Tomlinson, B.E.: Neurotransmitter enzyme abnormalities in senile dementia. J. Ncurol. Sci. 34, 247-265 (1977)

7 Perry, E.K., Tomlinson, B.E., Blessed, G., Bergmann, K., Gibson, P.H and Perry, R.H.: Correlation of cholinergic abnormalities with senile plaques and mental test scores in senile dementia. Br. Med. J. 2, $1457-1459$ (1978)

8 Wilcock, G.K., Esiri, M.M., Bowen, D.M. and Smith, C.C.T.: Alzheimer's disease; correlation of cortical choline acetyltransferase activity with severity of dementia and histological abnormalities. J. Neurol. Sci. 57, 407-417 (1982)

9 Altman, H.J., Crosl, R.D., Jenden, D.J. and Berman, R.D.: Further characterizations of the nature of the behavioral and neurochemical cffects of lesions to the nucleus basalis of Meynert in the rat. Neurobiol. Aging 4, $125-130$ (1985)

10) Friedman, E., Lerer, B. and Kuster, J.: Loss of cholinergic neurons in the rat neocortex produces deficits in passive avoidance learning. Pharmacol. Biochem. Behav. 19, $309-312$ (1983)

11 Hepler, D.J., Wenk, G.L., Cribbs, B.L., Olton, D.S. and Coylc, J.T.: Memory impairments following basal forebrain lesions. Brain Res. 346, 814 (1985)

12 Miyamoto, M., Kato, J., Narumi, S. and Nagaoka, A.: Characteristics of memory impairment following lesioning of the basal forebrain and medial septal nucleus in rats. Brain Res. 419, $19-$ 31 (1985)

13 Murray, C.L. and Fibiger, H.C.: Learning and memory deficits after lesions of the nucleus basalis magnocellularis: Reversal by physostigmine. Neuroscience 14, 1025-1031 (1985)

14 Ridley, R.M., Baker, H.F., Drewett, B. and Johnson, J.A.: Effects of ibotenic acid lesions of 
the basal forebrain on serial reversal learning in marmosets. Psychopharmacology (Berlin) 86, 438443 (1985)

15 Smith, G.: Animal models of Alzheimer's disease: experimental cholinergic denervation. Brain Res. Rev. 13, 103-118 (1988)

16 Segawa, M., Saito, H. and Nishiyama, N.: Alteration in choline acetyltransferase and tyrosine hydroxylase activities of various brain regions after passive avoidance performance in mice. Biogenic Amines 7, 191 - 197 (1990)

17 Hepler, D.J., Olton, D.S., Wenk, G.L. and Coyle, J.T.: Lesions in nucleus basalis magnocellularis and medial septal area of rats produce qualitatively similar memory impairments. J. Neurosci. 5, 866-873 (1985)

18 Flicker, C., Dean, R., Watkins, S., Fisher, S. and Bartus, R.: Behavioral and neurochemical effects following neurotoxic lesions of major cholinergic input to the cerebral cortex. Pharmacol. Biochem. Behav. 18, 973-981 (1983)

19 Lerer, B., Warner, J., Friedman, E., Vincent, G. and Gamzu, E.: Cortical cholinergic impairment and behavioral deficits produced by kainic acid lesions of rat magnocellular basal forebrain. Behav. Neurosci. 99, $661-677$ (1985)

20 Miyamoto, M., Shintani, M., Nagaoka, A. and Nagawa, Y.: Lesioning of the rat basal forebrain leads to memory impairments in passive and active avoidance tasks. Brain Res. 328, 97-104 (1985)

21 Bartus, R.T., Pontecorvo, M., Flicker, C., Dean, R.L. and Figneiredo, J.C.: Behavioral recovery following bilateral lesions of the nucleus basalis does not occur spontaneously. Pharmacol. Biochem. Behav. 24, $1287-1292$ (1986)

22 Dubois, B., Mayo, W., Agid, Y., Lemoal, M. and Simon, H.: Profound disturbances of spontaneous and learned behaviors following lesions of the nucleus basalis magnocellularis in the rat. Brain Res. 338, $249-258$ (1985)

23 Mesulam, M.-M., Mufson, E.J., Wainer, B.H. and Levey, A.I.: Central cholinergic pathways in the rat: An overvicw based on an alternative nomenclature (Ch1-Ch6). Neuroscience 10, 11851201 (1983)

24 Segawa, M., Saito, H. and Nishiyama, N.: Alteration in acetylcholine content and muscarinic cholinergic binding of various brain regions after performance of passive avoidance behaviors in mice. Biogenic Amines 7, 199 - 207 (1990)

25 Brashear, H.R., Zaborszky, L. and Heimer, L.: Distribution of GABAergic and cholinergic neurons in the rat diagonal band. Neuroscience 17 , $439-451$ (1986)

26 Fisher, R.S., Buchwald, N.A., Hull, C.D. and Levine, M.S.: GABAergic basal forebrain neurons profect to the neocortex: the localization of glutamic acid decarboxylase and choline acetyltransferase in feline corticopetal neurons. J. Comp. Neurol. 272, 489-502 (1988)

27 Walker, L.C., Koliatsos, V.E., Kitt, C.A., Richardson, R.T., Rökaeus, A. and Price, D.L.: Peptidergic neurons in the basal forebrain magnocellular complex of the rhesus monkey. $\mathbf{J}$. Comp. Neurol. 280, 272-282 (1989) 